\title{
Photobiodegradation of halogenated aromatic pollutants
}

\author{
Kushalatha Mutharasaiah ${ }^{1}$, Vidya Govindareddy ${ }^{2}$, Chandrakant Karigar ${ }^{3 *}$ \\ ${ }^{1}$ Department of Biotechnolgy, City College, Bangalore, India; \\ ${ }^{2}$ Department of Biochemistry and Chemistry, Government Science College, Bangalore, India; \\ ${ }^{3}$ Department of Biochemistry, Bangalore University, Bangalore, India. \\ Email: karigar@bub.ernet.in
}

Received 17 April 2010; revised 5 May 2010; accepted 7 May 2010.

\begin{abstract}
Release of wide range of compounds as a consequence of industrial development is now a serious environmental problem. Numerous hazardous waste sites have been generated worldwide resulting from the accumulation of xenobiotics in soil and water. Aromatic compounds constitute a large and diverse group of chemicals that are responsible for causing widespread environmental pollution. Among them halogenated aromatic hydrocarbons are very stable to undergo degradation due to resonance energy and inertness of carbon-halogen, carbon-hydrogen and carbon-carbon covalent bonds. The physico-chemical remedial strategies to clean up sites contaminated by these compounds are inadequate and economically inefficient. Therefore, research is increasingly being focused on development of biological approaches for their remediation. The hunt for the microorganisms degrading halogenated aromatic pollutants has been successful in discovering a diverse range of aerobic, anaerobic and phototrophic bacteria. The bacteria mineralize the toxic halogenated pollutants into harmless products thereby contributing towards conservation of the environment quality.
\end{abstract}

Keywords: Haloaromatics; Photometabolism; Dehalogenation; Phototrophic Bacteria

\section{INTRODUCTION}

\subsection{Halogenated Aromatic Pollutants Chlorinated Aromatic Pollutants}

Chlorinated aromatic compounds are major environmental pollutants because they are often released in substantial quantities, are toxic and resistant to degradation, and accumulate in sediment and biota. Although some compounds are degraded only slowly by soil and aquatic microorganisms, others are metabolized relatively quickly.

Some of the chlorinated aromatic compounds include chlorotoluene, chlorobenzenes chlorobenzoates, chlorophenols, 4-chlorophenylacetate and chlorophenoxyacetates [1]. Chlorobenzenes are used extensively as solvents, fumigants, and intermediates in the production of pesticides, dyes, disinfectants, room deodorants and moth control agents [2]. Chlorinated phenols are used as wood preservatives, herbicides, fungicides, and general biocides are a large group of toxic xenobiotics that are serious environmental pollutants [3-5].

Chlorinated derivatives of phenoxyacetates, such as Dichlorophenoxy acetic acid (2, 4-D) and 2,4,5-trichlorophenoxyacetic acid $(2,4,5-\mathrm{T})$, have been released into the environment as herbicides over the past 40 years [6]. Unlike many of the recalcitrant synthetic compounds, 2, 4-D is rapidly degraded by soil microorganisms [7].

\subsection{Brominated Aromatic Compounds}

Brominated aromatic compounds have found use as flame retardants. Fluorinated and iodinated aromatic compounds are components of pharmaceutical agents. The chemical inertness and hydrophobicity of many of these compounds has resulted in them becoming widely distributed in the environment; in particular accumulating in many terrestrial and aquatic organisms [8]. This coupled with their toxicity has given rise to concern about their fate in the environment. Some of the brominated persistent pollutants are 6-bromo-2,4,5-trichloro phenol (BrTriClP), pentabromophenol (PBP), 3,3',5,5' tetrabromobisphenol A(TBBPA), bromophenol (BP) and bromo benzoic acid (BBA). Brominated diphenyl ethers (BDEs), are environmentally persistent class of organic pollutants, in "biosolids" from four different regions of the United States. These compounds are widely used as flame retardants, and their presence suggests that the environmental consequences of land application of biosolids need further investigation. BDEs have been frequently detected in wild-caught fish, indicating another pathway for human exposure [4,9].

A diversity of natural aromatic brominated organic 
compounds can be found in a variety of biota, mostly aquatic species such as algae and sponges [10]. The Aplysina aerophoba is an example of a marine sponge in which such compounds can be found [11]. Two examples of natural aromatic brominated compounds are shown in Figure 1. These brominated compounds may function as a chemical defence against predators and biofouling (unwanted accretion of biota on the outside of a-wet-organism).

\subsection{Fluorinated Pollutants}

Hazardous chemicals that have entered the environment because they have leaked out from products during use and waste treatment include chlorinated compounds and brominated flame-retardants (BFRs). Now the alarm is being sounded for a completely new group of problematic micropollutants - the fluorinated hazardous substances [12]. They are used in many types of products to achieve a smooth surface that is preferably stain- and water-repellent. The fluorinated chemicals can be found in certain cleaning agents, paint and varnish, wax, floor polishing agents, impregnation agents for textiles, carpets, paper, furniture and shoes, fire-extinguishing liquids and photo paper.

In the following section, the phototrophic bacterial degradation of some halogenated aromatic compounds is discussed.

\section{PHOTOBIODEGRADATION}

Utilization of light energy by organisms for growth and survival is called phototrophy. The function of the anoxygenic photosynthetic apparatus is the transformation of light energy into an electrochemical gradient of protons across the photosynthetic membrane, which can be used for ATP production, active transport, motility, and other energy-consuming processes.

Aromatic compounds, whether from natural or synthetic sources are ubiquitous in most ecosystems. Purplenon sulphur photosynthetic bacteria are capable of degrading a wide variety of structurally diverse aromatic compounds anaerobically in the presence of light $[13,14]$. Among them Rhodopseudomonas palustris is perhaps the most nutritionally versatile. Members of Photosyn
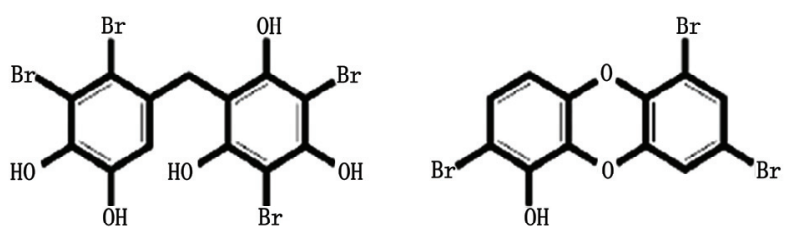

Figure 1. Examples of natural aromatic brominated organic compounds. The left compound is isolated from the red alga Rytiphlea tinctoria, the right one is isolated from the sponge Tedania ignis [10]. thetic anoxygenic bacteria include purple sulfur bacteria and green and purple nonsulfur bacteria. Anaerobic soil environments would provide favorable conditions for the proliferation of these bacteria [15]. In addition to cyanobacteria, these may contribute to the productivity through carbon fixation [16].

\subsection{Photobiodegradation of Halogenated Aromatic Compounds}

Among all the halogenated aromatic pollutants the photobiodegradation of chlorobenzoates are well studied. However the reports on the photobiodegradation of other halogenated aromatic compounds are scanty.

The degradation of chlorobenzoates by soil microorganisms have been reported by many researchers [1] and also the photobiodegradation of chlorobenzoates have been extensively studied in the phototrophic bacteria like Rhodopseudomonas polustris. During the metabolic study of 3-chlorobenzoate by a mixed phototrophic culture in the presence of benzoate, Rhodopseudomonas palustris WS17 was the dominant phototroph [17]. The anoxygenic photoheterotroph Rhodopseudomonas palustris DCP3, isolated by Van der Woude et al. [18] is the first example of an $R$. palustris strain that can use 3-chlorobenzoate (3CBA) as sole source of carbon under anoxic conditions in the presence of light. A unique property of this bacterium is that it does not need a cosubstrate for growth on the chlorinated compound, which is in contrast to previously described anaerobic phototrophic bacteria $[17,19]$. In an another degradation study, 3-chlorobenzoate was shown to be metabolized by Rhodopseudomonas palustris DCP3 under low-oxygen and phototropic conditions [20]. The phototrophic bacteria while metabolizing 3-chloro benzoate used the dechlorination pathway. In this the substrate 3-chlorobenzoate was dechlorinated to benzoate. If $R$. palustris grown on 3-chlorobenzoate, it can also use 2-chlorobenzoate, 4-chlorobenzoate or 3,5-dichlorobenzoate.

\subsection{Dehalogenation in Phototrophic Bacteria}

A few examples that phototrophic bacteria, including Rhodospirillum and Rhodopseudomonas sp., can grow phototrophically under anaerobic conditions using halocarboxylic acids or 3-chlorobenzoate have been reported [3]. We are currently investigating the possible role of Pseudomonas stutzeri in metabolizing 2-chlorophenol and 4-chlorophenol under phototropic and denitrification conditions.

\section{CONCLUSIONS}

Purple-non sulphur photosynthetic bacteria are capable of degrading a wide variety of structurally diverse aromatic compounds anaerobically in the presence of light. 
Anaerobic soil environments would provide favorable conditions for the proliferation of these bacteria. Anaerobic processes are beneficial for eliminating pollutants from contaminated sites, in which oxygen is often unavailable due to its quick depletion with easily utilizable substrates, low solubility in water and low rate of transportation in saturated porous matrices such as soils and sediments. The metabolism of haloaromatics by anaerobic phototrophic bacteria is made possible by dislodging carbon-halogen bond by dechlorination or halorepiration, denitrifying conditions or under meth-anogenic environments. It is hoped that anaerobic and phototropic microorganisms are better suited than aerobes for the remediation of natural halogenated aromatics and pollutants arising from industrial applications and effluents.

\section{REFERENCES}

[1] Chaudhry, G.R. and Chapalamadugu, S. (1991) Biodegradation of Halogenated Organic Compounds. Microbiological Reviews, 55(1), 59-79.

[2] Bhatt, P., Kumar, M.S., Mudliar, S. and Chakrabarti, T. (2007) Biodegradation of chlorinated compounds-A review. Critical Reviews in Environmental Science and Technology, 37(2), 165-198.

[3] Thakur, I.S. (2007) Environmental microbiology xenobiotics: Pollutants and their degradation-methane, benzene, pesticides, bioabsorption of metals. http://hdl.handle.net/ $123456789 / 664$

[4] Stanlake, G.J. and Finn, R.K. (1982) Isolation and characterization of a pentachlorophenol-degrading bacterium. Applied and Environmental Microbiology, 44(6), 14211427.

[5] Steiert, J.G. and Crawford, R.L. (1985) Microbial degradation of chlorinated phenols. Trends in Biotechnology, 3(12), 300-305.

[6] Karns, J.S., Kilbane, J.J., Duttagupta, S. and Chakrabarty, A.M. (1983) Metabolism of halophenols by 2,4,5-trichlorophenoxyacetic acid-degrading Pseudomonas cepacia. Applied and Environmental Microbiology, 46(5), 1176-1181.

[7] Kilpi, S., Backstrom, V. and Korhola, M. (1980) Degradation of 2-methyl-4 chlorophenoxyacetic acid (MCPA), 2,4-dichlorophenoxyacetic acid (2,4-D), benzoic acid and salicylic acid by Pseudomonas sp. HV3. FEMS Microbiology Letters, 8, 177-182.

[8] Commandeur, L.C.M. and Parsons, J.R. (1990) Degradation of halogenated aromatic compounds. Biodegrada- tion, 1(2-3), 207-220.

[9] Hale, R.C., La Guardia, M.J., Harvey, E.P., Gaylor, M.O., Mainor, T.M. and Duff, W.H. (2001) Flame retardants: Persistent pollutants in land-applied sludges. Nature, 412(6843), 140-141.

[10] Gribble, G.W. (1999) The diversity of naturally occurring organobromine compounds. Chemical Society Reviews, 28(5), 335-346.

[11] Ahn, Y.B., et al. (2003) Reductive dehalogenation of brominated phenolic compounds by microorganisms associated with the marine sponge Aplysina aerophoba. Applied and Environmental Microbiology, 69(7), 41594166.

[12] (2006) Fluorinated pollutants in all-weather clothing. Friends of the Earth Norway - Report 2.

[13] Sikdar, S.K. and Irvine, R.L. (1997) Biodegradation technology developments (Bioremediation Vol 2. princeples and practice). Technomic Publ. Co, Lancaster.

[14] Gottschal, J.C. (1994) Anaerobic degradation of halogenated benzoic acids by photoheterotrophic bacteria. FEMS Microbiology Letters, 119(1-2), 199-208.

[15] Bashan, Y. and Holguin, G. (2002) Plant growth-promoting bacteria: A potential tool for arid mangrove reforestation Trees. Trees-Structure and Function, 16(2-3), 159-166.

[16] Day, J.W., Jr., Hall, C.A.S., Kemp, W.M., Yanez-Arancibia, A. and Christian, R.R. (Eds.) (1989) Microbial ecology and organic detritus in estuaries. Estuarine Ecology, Wiley, New York, 257-308.

[17] Kamal, V.S. and Wyndham, R.C. (1990) Anaerobic phototrophic metabolism of 3-chlorobenzoate by Rhodopseudomonas palustris WS17. Applied and Environmental Microbiology, 56(12), 3871-3873.

[18] Van der Woude, B.J., De Boer, M., Van der Put, N.M., Van der Geld, J.F.M., Prins, R.A. and Gottschal, J.C. (1994) Anaerobic degradation of halogenated benzoic acids by photoheterotrophic bacteria. FEMS Microbiology Letters, 119(1-2), 199-208.

[19] Blasco, R. and Castillo, F. (1992) Light-dependent degradation of nitrophenols by the phototrophic bacterium Rhodobacter capsulatus E1F1. Applied Environmental Microbiology, 58, 690-695.

[20] Krooneman, J., Akker, S.V.D., Pedro Gomes, T.M., Forney, L.J. and Gottschal, J.C. (1999) Degradation of 3-chlorobenzoate under low-oxygen conditions in pure and mixed cultures of the anoxygenic photoheterotroph Rhodopseudomonas palustris DCP3 and an Aerobic Alcaligenes species. Applied and Environmental Microbiology, 65(1), 131-137. 